\title{
Новые возможности в лечении метастатического РГЖ в постменопаузе: клинический обзор ключевых исследований
}

Получено: 26.07.2019

Принято в печать: 31.07.2019

DOI: $10.32471 /$ clinicaloncology.2663-466X.39.22815

\begin{abstract}
За последние несколько десятилетий появилось множество видов эндокринной терапии первой линии для пациенток c ER+/PgR+ распространенным и/или метастатическим раком грудной железы (РГЖ). Рекомендации относительно выбора терапии первой линии для женщин в период постменопаузы с местно-распространенным или метастатическим PГЖ, ER+/PgR+, включают эндокринную терапию ингибитором ароматазы третьего поколения (анастрозол, летрозол или экземестан) или тамоксифеном [1-4]. Известно, что при гормон-рецептор-положительном РГЖ ингибиторы ароматазы третьего поколения обладают более высокой эффективностью по сравнению с тамоксифеном относительно времени до прогрессирования [5-9]. В то же время высокая распространенность резистентности к лечению ингибиторами ароматазы свидетельствует об актуальности разработки дополнительных вариантов терапии с различными механизмами действия. Одним из вариантов, предоставляющих дополнительные возможности терапии, является фулвестрант (Фазлодекс), продемонстрировавший высокую эффективность наравне с приемлемым профилем безопасности.
\end{abstract}

Ключевые слова: гормон-рецептор-положительный распространенный и/или метастатический рак грудной железы; ингибиторы ароматазы; анастрозол; фулвестрант; Фазлодекс.

Рост и пролиферация опухолевых клеток гормонзависимого РГЖ зависит от активности рецепторов эстрогенов (oestrogen receptors - ER) - ER $\alpha$ и ER $\beta$, которые в норме активируются эстрогенами. После связывания с гормоном ER подвергаются димеризации и транслоцируются в клеточное ядро. Затем димеры связываются с ДНК в регуляторных областях целевых генов, влияя таким образом на интенсивность их экспрессии. Молекула ER $\alpha$ состоит из нескольких доменов: лигандсвязывающий домен (связывающий $17 \beta$-эстрадиол), ДНК-связывающий домен, шарнирный или димеризационный домен и два трансактивирующих домена - AF (activation function)-1 и AF-2. AF-1 - домен с транскрипционной активаторной функцией находится на $\mathrm{N}$-концевом участке рецептора, ответственном за белок-белковые взаимодействия и транскрипционную активацию гена-мишени независимо от связывания с лигандом [10-12]. Лигандсвязывающий домен содержит участок с активационной функцией $\mathrm{AF}-2$, зависимой от присутствия лиганда. Он ответственен за конформационные изменения ER при наличии лигандов и последующее связывание димеров ER с коактиваторами и корепрессорами в зависимости от связывания с лигандом. При связывании лиганда с рецептором происходит его димеризация, ядерная транслокация и трансактивация экспрессии генов-мишеней.

$\mathrm{ER} \alpha$ имеет разные механизмы действия:

1) функционирует как фактор транскрипции: активирует специфические элементы ответа на эстрогены (oestrogen response elements - ERE) в промоторах генов-мишеней;

2) связывание и активация других транскрипционных факторов;

3) активация независимым от лиганда способом посредством перекрестного взаимодействия с тирозинкиназными путями;

4) оказание «негеномного» действия, при котором эстрогены активируют мембраносвязанные и связанные с G-белком ER (GPER, также известный как GPR30) и запускают нисходящий сигнальный путь.

Управление действиями ER включает рекрутирование корегуляторов AF-доменом. В то время как AF-2 активируется эстрогеном в лигандзависимых процессах, AF1 активируется факторами роста посредством активации митоген-активируемой протеинкиназы (mitogen-activated protein kinase MAPK) $[13,14]$. Полная агонистическая функция рецептора возможна благодаря наличию обеих активностей.

\section{МЕХАНИЗМ ДЕЙСТВИЯ И ПРЕИМУЩЕСТВА} ПРИМЕНЕНИЯ ФУЛВЕСТРАНТА (ФАЗЛОДЕКС)

Фулвестрант - аналог 17-эстрадиола, является селективным деградатором ER, который блокирует функцию ER [15-17]. Поскольку димеризация рецептора блокируется, это вызывает ускоренную деградацию и отрицательную регуляцию ER [16].

Механизм действия тамоксифена, его метаболитов и эстрогена похожи, а разница основана на дезактивации AF-2 в комплексе тамоксифен-эстрогеновый рецептор (TAM-ER). Частичная агонистическая активность тамоксифена является результатом действия AF-1 и зависит от типа клеток и промотора, с которым связан транскрипционный комплекс. Таким образом, тамоксифен частично выключает только активный домен AF-2. В свою очередь, фулвестрант блокирует оба активных домена, практически полностью выключая активность рецептора и приводя к его ускоренному разрушению. Фулвестрант связывается с ER $\alpha$, конкурируя при этом с эстрадиолом за сайт связывания. Он характеризуется высокой аффинностью связывания, которая составляет $89 \%$ таковой для эстрадиола, что намного больше, чем сродство тамоксифена, которое составляет 2,5\% [16]. Связывание фулвестранта с ER $\alpha$ препятствует димеризации ER, ингибирует ядерную транслокацию ER и активацию и способствует деградации ER (отрицательная регуляция ER), приводя к полному ингибированию передачи сигналов ER $\alpha$. Блокируя как AF-1, так и AF-2 (в то время как селективные модуляторы ER ингибируют только AF-2), фулвестрант является селективным деградатором рецептров эстрогена, лишенным какой-либо агонистической активности, включая влияние на эндометрий [18-21].

Связывание комплекса фулвестрант-ER c ERE ингибируется, как и функции AF-1 и AF-2, а также связывание коактиватора. Повышенная деградация ER, опосредованная фулвестрантом, возможно, основана на прекращении активации ER другими медиаторами, например дофамином, циклическим аденозин 3, 5'-монофосфатом (цАМФ) или факторами роста [22-28].

Более того, в отличие от тамоксифена, фулвестрант является селективным деградатором ER, не обладает агонистоподобной активностью, о чем свидетельствуют исследования, оценивающие утеротрофические эффекты препарата у животных и женщин в период постменопаузы с раком грудной железы, 
а также снижение экспрессии рецептора прогестерона (белка, регулируемого эстрогенами), наблюдаемого при применении фулвестрантом [29-35]. Указанные различия в механизме действия селективных модуляторов ER (selective oestrogen receptor modulators - SERM), к которым принадлежит тамоксифен, и фулвестранта объясняют отсутствие усиления активности роста эндометрия при применении последнего, что отмечалось в ходе доклинических исследований [22].

Фулвестрант также оказывает незначительное влияние на сывороточные уровни фолликулостимулирующего гормона и лютеинизирующего гормона у женщин в период постменопаузы с раком грудной железы [36, 37], что свидетельствует об отсутствии клинически значимых периферических стероидных эффектов [38]. Более того, при применении фулвестранта у пациентов с раком грудной железы не было отмечено клинически значимого изменения уровней маркеров костного обмена в сыворотке крови $[39,40]$.

Таким образом, фулвестрант селективно связывается с ER, вызывая их деградацию в опухолевых клетках РГЖ и делая рецепторы недоступными для эстрогена и подавляя как эстрогензависимые, так и эстрогеннезависимые сигнальные пути (AF-1 и AF-2), что приводит к полному подавлению сигнального пути эстрогена через ER, в отличие от селективных модуляторов ER. Кроме того, фулвестрант связывается с ER с более высокой аффинностью, чем тамоксифен, и не проявляет агонистической активности.

Первично фулвестрант был одобрен как препарат второй линии, однако успешные результаты его широкого применения дали основания для проведения клинических исследований, которые продемонстрировали его преимущества в качестве первой линии терапии. Сегодня монотерапия фулвестрантом, в соответствии с современными руководствами по лечению $\mathrm{ER}+/ \mathrm{PgR}+$ распространенного рака грудной железы у женщин в период постменопаузы, рекомендована в качестве первой линии эндокринной терапии наряду с другими вариантами, включая ингибиторы ароматазы (например анастрозол, летрозол, экземестан), селективные модуляторы ER (например тамоксифен) и пр. [41-43]. Выбор наиболее подходящего режима основывается на анализе ряда факторов, таких как тип, продолжительность и время после адъювантной эндокринной терапии (если таковая была), локализация метастазов (например кости, мягкие ткани, внутренние органы), сопутствующие заболевания и предпочтения пациента [42, 44]. Кроме того, следует учитывать, что ежемесячные инъекции фулвестранта по сравнению с ежедневным пероральным приемом, необходимым для других эндокринных терапий, могут способствовать повышению приверженности пациентов лечению [45]. Фулвестрант также может быть приемлемым вариантом для пациентов, испытывающих сложности с пероральным приемом лекарственных средств. Монотерапия фулвестрантом в качестве эндокринного лечения первой линии при распространенном раке грудной железы непосредственно не сравнивалась с другими эндокринными агентами, кроме анастрозола, однако, согласно результатам ряда метаанализов, фулвестрант демонстрирует преимущества по сравнению с несколькими такими компараторами [46, 47]. В одном из таких метаанализов [46] фулвестрант ассоциировался с лучшей выживаемостью без прогрессирования (ВБП), чем анастрозол, экземестан, тамоксифен и торемифен, и имел преимущества по сравнению с анастрозолом, экземестаном и тамоксифеном. Аналогичным образом другой анализ [47] доказал преимущество фулвестранта 500 мг в эффективности по показателю времени до прогрессирования заболевания (ВДП)/ВБП, за которым следует летрозол, анастрозол, экземестан и тамоксифен. Кроме того, поскольку лечение рака грудной железы часто является паллиативным, минимизация токсичности - также важный фактор при выборе подходящего препарата [44]. Так, фулвестрант, как правило, хорошо переносился и де- монстрировал низкую частоту серьезных побочных реакций, связанных с лечением.

Таким образом, Фазлодекс (фулвестрант) показан для лечения рака молочной железы с ER+, местно-распространенного или с метастазами у женщин в период постменопаузы: ранее не получавших гормональную терапию; в случае рецидива болезни во время или после адъювантной антиэстрогенной терапии или прогрессирования заболевания при антиэстрогенной терапии [48].

Доза фулвестранта 500 мг была одобрена на основе данных клинического исследования III фазы, двойного слепого "Comparison of Faslodex in Recurrent or Metastatic Breast Cancer» (CONFIRM) [49], в котором сравнивали фулвестрант 500 мг и фулвестрант 250 мг у пациентов с ER+/PgR + pacпространенным РГЖ, у которых прогрессирование заболевания наступило на фоне эндокринной терапии. В исследовании CONFIRM было показано, что ВБП и общая выживаемость (OВ) были больше для фулвестранта 500 мг по сравнению с таковым в дозе 250 мг. Далее будет рассмотрен ряд исследований, которые демонстрируют преимущество фулвестранта 500 мг относительно эффективности терапии как по сравнению с фулвестрантом 250 мг, так и по сравнению с ведущим представителем класса ингибиторов ароматазы наравне с приемлемым профилем безопасности и переносимостью [50-52].

\section{ИССЛЕДОВАНИЕ CONFIRM: \\ ФАЗЛОДЕКС 500 УВЕЛИЧИВАЕТ ОБЩУЮ ВЫЖИВАЕМОСТЬ}

Известно, что фулвестрант снижает уровень ER в клетках дозозависимым образом [16, 53, 54]. В то же время более ранние клинические исследования эффективности применения фулвестранта 250 мг по сравнению с анастрозолом у пациентов с гормонзависимым РГЖ в период постменопаузы, которые предварительно получали тамоксифен, показали, что оба препарата характеризуются одинаковой эффективностью и приемлемым профилем безопасности с низкой частотой отмены препарата [34, 36]. Анализ результатов упомянутых исследований позволил предположить, что повышение дозы фулвестранта (Фазлодекс) позволит повысить эффективность лечения [34-37, 54, 55], это и было показано в ходе исследования CONFIRM [49].

CONFIRM - это двойное слепое многоцентровое клиническое исследование III фазы с параллельными группами, в котором оценивали эффективность двух различных доз фулвестранта - одобренной на тот момент (250 мг каждые 28 сут) и более высокой (500 мг на 0-, 14- и 28-е сутки и каждые 28 сут после этого). В исследовании принимали участие женщины c ER+ распространенным РГЖ, у которых было отмечено прогрессирование заболевания после ранее назначенного лечения. Первичной конечной точкой исследования была выбрана ВБП.

Согласно полученным результатам, фулвестрант 500 мг значительно продлевает ВБП по сравнению с фулвестрантом 250 мг (относительный риск (ОР) 0,$80 ; 95 \%$ доверительный интервал (ДИ) $0,68-0,94 ; p=0,006$ ) (рис. 1). Медиана времени ВБП составляла 6,5 и 5,5 мес в группах, получавших 500 и 250 мг фулвестранта соответственно. Через 12 мес 34 и 25\% пациентов, получавших данный препарат в дозе 500 и 250 мг соответственно, остались живы и у них не было отмечено прогрессирования заболевания. Аналогичные показатели составили 16 и $11 \%$ соответственно через 24 мес лечения.

Медиана продолжительности клинического эффекта составила 16,6 и 13,9 мес в группах, получавших фулвестрант в дозе 500 и 250 мг соответственно. На момент первичного анализа ОВ (50\% пациентов оставались живыми) медиана времени до смерти составляла 25,1 и 22,8 мес для пациентов, получавших фулвестрант в дозе 500 и 250 мг соответственно (ОР 0,84; 95\% ДИ 0,69-1,03; $\mathrm{p}=0,091$ ) (рис. 2).

По информации обновленного анализа данных исследования CONFIRM (25\% пациентов оставались живы), медиана 


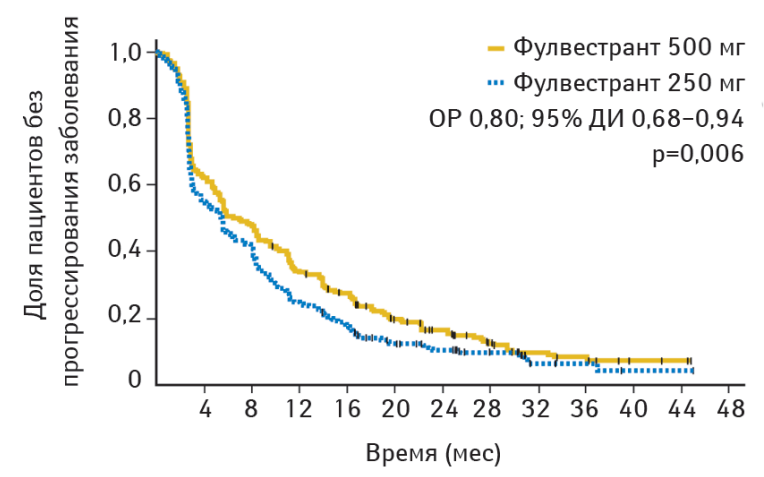

Количество пациентов, оставшихся в живых

Фулвестрант 500 мг 36221616311390543719127320 Фулвестрант 250 мг $374199144 \quad 85 \quad 60352512 \quad 4 \quad 31110$

Рис. 1. ВБП в группах пациентов, получавших фулвестрант в дозе 250 и 500 мг

ОВ при применении фулвестранта в дозе 500 мг на 4,1 мес больше, чем при применении фулвестранта в дозе 250 мг (26,4 мес в сравнении с 22,3 мес соответственно, ОР 0,$81 ; 95 \%$ ДИ 0,69-0,96; номинальный $\mathrm{p}=0,016$ ) [56].

При этом профили безопасности применения фулвестранта в дозе 500 и 250 мг были сопоставимыми [49].

Таким образом, эти результаты демонстрируют, что режим применения фулвестранта 500 мг внутримышечно (на 0-, 14и 28 -е сутки и каждые 28 сут после этого) более целесообразен с позиций клинической эффективности в медицинской практике по сравнению с фулвестрантом в дозе 250 мг. Благодаря тому что фулвестрант подтвердил эффективность в отношении повышения показателей OB в исследовании CONFIRM, пациенты с распространенным РГЖ имеют более высокие шансы прожить дольше, получая фулвестрант во 2- или 3-й линии после тамоксифена.

\section{ИССЛЕДОВАНИЕ FIRST: ПРЕИМУЩЕСТВА ПРИМЕНЕНИЯ ФАЗЛОДЕКСА ПО СРАВНЕНИЮ С ИНГИБИТОРОМ АРОМАТАЗЫ ТРЕТЬЕГО ПОКОЛЕНИЯ}

Клиническая эффективность фулвестранта была первоначально продемонстрирована в двух исследованиях III фазы, в которых сравнивали фулвестрант в дозе 250 мг/мес с анастрозолом по 1 мг/сут в качестве второй линии терапии распространенного РГЖ [34, 36]. Комбинированный анализ этих исследований показал, что время до прогрессирования (ВДП) для пациентов, получавших фулвестрант в дозе 250 мг, не уступало таковому в группе, получавшей анастрозол [35]. Таким образом, после успешного проведения исследования CONFIRM логичным было сравнить эффективность фулвестранта в повышенной дозе 500 мг с анастрозолом. Именно такая цель была поставлена при проведении исследования «Fulvestrant First-Line Study Comparing Endocrine Treatments» (FIRST).

FIRST - рандомизированное открытое многоцентровое исследование II фазы, в котором сравнивали эффективность и профиль безопасности фулвестранта в дозе 500 мг (на 0-, 14-, 28-е сутки и каждые 28 сут после этого) с анастрозолом 1 мг (ежедневно) в качестве первой линии терапии у женщин в период постменопаузы с ER+/PgR+ местно-распространенным/метастатическим РГЖ [52].

Ключевыми критериями включения в исследование были: женщины в период постменопаузы с ER+ или рецептор прогестерона (progesterone receptor - PgR) положительным местно-распространенным или метастатическим РГЖ, ранее не получавшие эндокринную терапию или получавшие эндокринную терапию за >12 мес до рандомизации по поводу ранних стадий РГЖ. Основными критериями исключения были: наличие метастазов, угрожающих жизни, и предшествующее

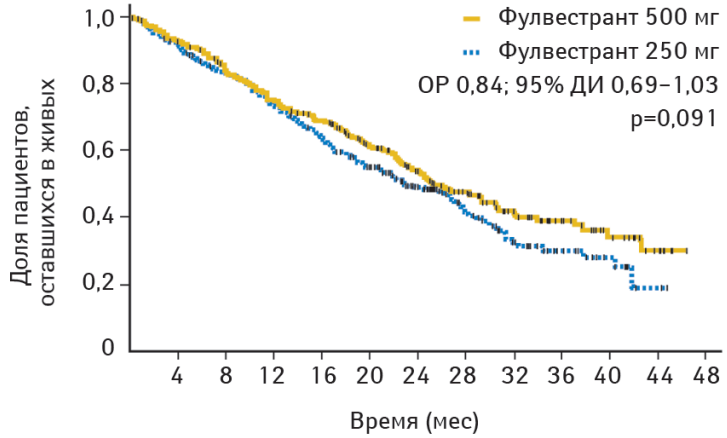

Количество пациентов, оставшихся в живых

Фулвестрант 500 мг 3623302852512231651167446291660 Фулвестрант 250 мг 3743382992602221571076134181020

Рис. 2. ОВ в группах пациентов, получавших фулвестрант в дозе 250 и 500 мг

лечение неодобренным препаратом. В качестве первичной конечной точки были выбраны частота клинического эффекта и медиана ВДП.

В общей сложности 205 пациентов были случайным образом распределены для получения фулвестранта 500 мг $(\mathrm{n}=102)$ или анастрозола 1 мг $(\mathrm{n}=103)$ в 62 центрах 9 стран (Бразилия, Болгария, Чешская Республика, Франция, Италия, Польша, Испания, США и Великобритания). Базовые и демографические характеристики пациентов в группах лечения были схожими [50]. Доля пациентов, которые ранее не получали эндокринную терапию на ранних стадиях заболевания, была одинаковой для участников, получавших фулвестрант в дозе 500 мг и анастрозол $(71,6$ и 77,7\% пациентов в начале исследования соответственно). Среди тех, кто ранее получал терапию, большинству назначался тамоксифен.

Первый анализ был выполнен, когда 79,5\% пациентов прекратили исследуемое лечение [51]. На момент первого анализа только у $36 \%$ пациентов отмечалось прогрессирование заболевания и медиана ВДП не была достигнута для фулвестранта. Согласно полученным результатам, при применении фулвестранта ВДП увеличивалось более чем на 10 мес по сравнению с применением анастрозола. Так, медиана ВДП составила 23,4 мес для фулвестранта против 13,1 мес для анастрозола, а снижение риска прогрессирования составило $34 \%$ (ОР 0,66; $95 \%$ ДИ $0,47-0,92 ; \mathrm{p}=0,01)$. Частота клинического эффекта составила 72,5 и $67,0 \%$ для фулвестранта в дозе 500 мг и анастрозола соответственно.

Оценка показателя ОВ изначально не была запланирована в протоколе, и впоследствии в него были внесены поправки для оценки ОВ после того, как в живых осталось около $35 \%$ пациентов. Следует отметить, что не все больные участвовали в дальнейшем наблюдении для оценки ОВ. Влияние лечения на ОВ было изучено в нескольких подгруппах.

На момент проведения последующего анализа OВ 63 из 102 пациентов в группе фулвестранта в дозе 500 мг $(61,8 \%)$ и 74 из 103 пациентов в группе анастрозола $(71,8 \%)$ умерли. Показатели первичного анализа ОВ улучшились в группе фулвестранта в дозе 500 мг по сравнению с анастрозолом 1 мг; ОР составил 0,70 (95\% ДИ 0,50-0,98; логранговый критерий $\mathrm{p}=0,04$; медиана ОВ $-54,1$ мес против 48,4 мес; рис. 3). Было установлено, что ОВ для фулвестранта в дозе 500 мг по сравнению с анастрозолом в целом совпадает во всех подгруппах.

Большинство побочных реакций были расценены исследователями как не связанные с лечением. Были зарегистрированы две побочные реакции, ассоциированные с терапией (один случай артериальной гипертензии и один случай легочной эмболии, оба в группе лечения фулвестрантом в дозе 500 мг). 


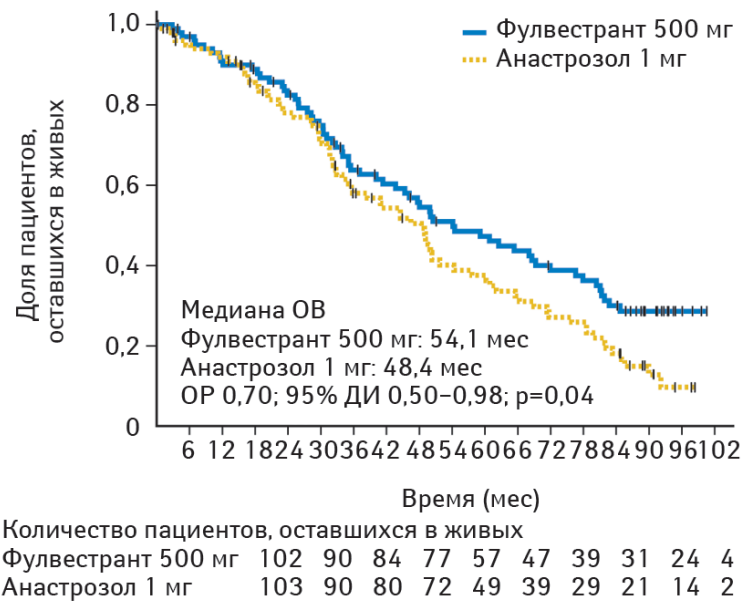

Рис. 3. ОВ в группах пациентов, получавших фулвестрант или анастрозол

Таким образом, в исследовании FIRST показано улучшение ОВ при лечении фулвестрантом в дозе 500 мг по сравнению с анастрозолом в качестве первой линии терапии при ER+ распространенном РГЖ, со снижением риска смерти приблизительно на $30 \%$. Так, улучшение ВДП привело к улучшению ОВ примерно на 6 мес при применении фулвестранта в дозе 500 мг $(54,1 \mathrm{меc)}$ по сравнению с анастрозолом $(48,4$ мес), что согласуется с данными относительно улучшения OB при применении фулвестранта в дозе 500 мг по сравнению с 250 мг во второй линии терапии, согласно данным, полученным в исследовании CONFIRM [57]. Кроме того, не было зарегистрировано каких-либо новых особенностей профиля безопасности или проблем переносимости на этапе наблюдения в этом исследовании, что согласуется с ранее полученными данными о безопасности [50, 51]. Улучшение ОВ при применении фулвестранта в дозе 500 мг $(54,1$ мес) по сравнению с анастрозолом (48,4 мес) наблюдалось даже несмотря на то, что медиана ОВ для группы, получавшей анастрозол, в этом исследовании была выше, чем сообщалось ранее. Например, ОВ при применении анастрозола в качестве эндокринной терапии первой линии при распространенном РГЖ в комбинированном анализе двух исследований III фазы составила 39,2 мес [58], а при монотерапии анастрозолом в комбинированном исследовании III фазы - 41,3 мес [59]. Кроме того, соответствующие средние значения ОВ 34,0 мес (летрозол) [8] и 37,2 мес (экземестан) [36] были зарегистрированы для других ингибиторов ароматазы. Поэтому маловероятно, что в настоящем анализе переоценивается степень улучшения выживаемости при применении фулвестранта в дозе 500 мг по сравнению с анастрозолом, что могло бы быть возможным, если бы лечение в контрольной группе (анастрозол) было бы недостаточно эффективным. Таким образом, применение фулвестранта в дозе 500 мг связано с улучшением OB по сравнению с анастрозолом в первой линии терапии ER+ распространенного РГЖ. Так, впервые было продемонстрировано, что эндокринная монотерапия обладает более высокой эффективностью по сравнению с ингибиторами ароматазы третьего поколения.

\section{FALCON: ФУЛВЕСТРАНТ УВЕЛИЧИВАЕТ ВБП ЕЩЕ НА 2,8 МЕС ПО СРАВНЕНИЮ \\ С ИНГИБИТОРАМИ АРОМАТАЗЫ}

Исследование «Fulvestrant and Anastrozole Compared in Hormonal Therapy Naive Advanced Breast Cancer» (FALCON) было разработано после получения положительных результатов в ходе II фазы исследования FIRST [60]. Дизайн исследования исключал возможные источники систематической ошибки, такие как применявшаяся ранее эндокринная терапия, в результате которой может развиться приобретенная эндокринная резистентность. Целью исследования FALCON стало сравнить эффективность фулвестранта и анастрозола по показателю ВБП и избежать при этом влияния на результат предшествующей эндокринной или гормональной терапии.

FALCON - рандомизированное двойное слепое клиническое исследование III фазы, в котором приняли участие более 100 медицинских центров в 20 странах. В исследование FALCON были включены женщины в период постменопаузы c ER+ и/или $\mathrm{PgR}+$ местно-распространенным или метастатическим РГЖ, которые ранее не получали эндокринную терапию. Это было сделано с целью избежать снижения эффективности в контрольной группе путем воздействия адъювантной эндокринной терапии. Первичной конечной точкой была выбрана ВБП.

В исследование было включено 524 пациента, из них 462 были рандомизированы на две группы: 230 пациентов получали фулвестрант и 232 - анастрозол.

На момент анализа полученных данных было зарегистрировано 309 случаев прогрессирования заболевания, из них $143(62 \%)$ из 230 имели место в группе, получавшей фулвестрант, а 166 (72\%) из 232 - в группе анастрозола. Применение фулвестранта ассоциировалось со статистически значимым улучшением ВБП по сравнению с анастрозолом (ОР 0,797; $95 \%$ ДИ 0,637-0,999; p=0,0486; рис. 4). Медиана ВБП составила 16,6 мес (95\% ДИ 13,83-20,99) при применении фулвестранта и 13,8 мес $(11,99-16,59)$ в группе, получавшей анастрозол (разница 2,8 мес).

Средняя продолжительность ответа на лечение была больше в группе, получавшей фулвестрант (20,0 мес, 95\% ДИ 15,90-27,63), по сравнению с группой, получавшей анастрозол (13,2 мес, 95\% ДИ 10,64-16,72).

Частота клинического эффекта составила 78\% (180/230) при применении фулвестранта и 74\% (172/232) - в группе, получавшей анастрозол (ОР 1,25; 95\% ДИ 0,82-1,93; $\mathrm{p}=0,3045)$. Медиана продолжительности клинического эффекта составила 22,1 мес (95\% ДИ 18,46-24,97) при применении фулвестранта и 19,1 мес $(16,53-20,47)$ в группе, получавшей анастрозол. OB в исследовании FALCON была вторичной конечной точкой. На момент проведения анализа медиану ОВ невозможно было рассчитать из-за недостаточного времени наблюдения (31\% зрелости данных).

Эффективность лечения в отношении ВБП была в основном одинаковой во всех подгруппах пациентов, за исключением некоторых из них.

О серьезных побочных эффектах сообщили 30 (13\%) из 228 пациентов, получавших фулвестрант, и 31 (13\%) из 232 пациентов, которым был назначен анастрозол. Неблагоприятные явления 3-й степени или выше были зарегистриро-

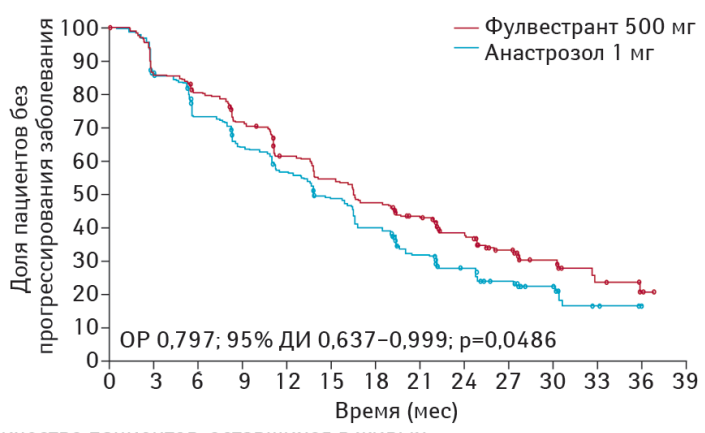

Количество пациентов, оставшихся в живых

Фулвестрант 500 мг 23018717115012411096816344241120 Анастрозол 1 мг 23219416213912010284604531221000

Рис. 4. ВБП в группах пациентов, получавших фулвестрант или анастрозол 
ваны у 51 (22\%) из 228 пациентов, получавших фулвестрант, и у 41 (18\%) из 232 пациентов группы анастрозола.

Первичная конечная точка этого исследования была достигнута: ВБП пациентов, получавших фулвестрант, значительно превышает таковую у пациентов из группы анастрозола, подтверждая гипотезу о том, что фулвестрант является более эффективным препаратом для терапии, чем анастрозол, у женщин в период постменопаузы с ER+/PgR+ местно-распространенным или метастатическим РГЖ, которые ранее не получали эндокринную терапию. Фулвестрант обеспечивает дополнительное увеличение медианы ВБП по сравнению с ингибитором ароматазы анастрозолом еще на 2,8 мес у пациентов с ER+ распространенным РГЖ в период постменопаузы. При этом показатель ОР для ВБП, полученный в этом исследовании $(0,797)$, аналогичен улучшению, продемонстрированному ингибиторами ароматазы третьего поколения по сравнению с тамоксифеном [5-8]. Оба препарата обладают сопоставимым профилем безопасности и переносимостью.

\section{В ЗАКЛЮЧЕНИЕ}

Приведенные выше исследования обеспечивают прямое сравнение терапевтической эффективности селективного деградатора ER фулвестранта и ингибитора ароматазы третьего поколения, в том числе без влияния предыдущего воздействия адъювантной эндокринной терапии любого типа, доказывая преимущества Фазлодекса (фулвестранта) относительно эффективности лечения, превышающей таковую для ингибиторов ароматазы третьего поколения, и демонстрируя приемлемый профиль безопасности. Тем самым Фазлодекс открывает новые возможности эффективной терапии $\mathrm{ER}+/ \mathrm{PgR}+$ местнораспространенного или метастатического РГЖ у пациентов в период постменопаузы.

\section{СПИСОК ИСПОЛЬЗОВАННОЙ ЛИТЕРАТУРЫ}

1. National Comprehensive Cancer Network. (2016). NCCN Clinical Practice Guidelines in Oncology Version.2.2015: Breast Cancer. 2015. Retrieved from http://www.nccn.org/professionals/physician_gls/PDF/breast.pdf.

2. Rugo, H. S., Rumble, R. B., Macrae, E., Barton, D. L., Connolly, H. K., Dickler, M. N., ...\& Johnston, S. R. (2016). Endocrine therapy for hormone receptorpositive metastatic breast cancer: American Society of Clinical Oncology Guideline. Journal of Clinical Oncology, 34(25), 3069-3103. doi: 10.1200/JCO.2016.67.1487.

3. Cardoso, F., Costa, A., Norton, L., Senkus, E., Aapro, M., Andre, F. .\& Cardoso, M. J. (2014). ESO-ESMO 2nd international consensus guidelines for advanced breast cancer (ABC2). Annals of oncology, 25(10), 1871-1888 doi: 10.1093/annonc/mdu385.

4. Burstein, H. J., Temin, S., Anderson, H., Buchholz, T. A., Davidson, N. E., Gelmon K. E., ...\& Griggs, J. J. (2014) Adjuvant endocrine therapy for women with hormone receptor-positive breast cancer: American Society of Clinical Oncology Clinica Practice Guideline Focused Update. Journal of Clinical Oncology, 32: 2255-2269. doi: $10.1200 / J C O .2013 .54 .2258$

5. Nabholtz, J. M., Buzdar, A., Pollak, M., Harwin, W., Burton, G., Mangalik, A., ... \& Von Euler, M. (2000). Anastrozole is superior to tamoxifen as first-line therapy for advanced breast cancer in postmenopausal women: results of a North American multicenter randomized trial. Journal of Clinical Oncology, 18(22), 3758-3767. Retrieved from https://www.mcgill.ca/pollak-lab/files/pollak-lab/anastrazole is superior_to tamoxifen_as_first-line_therapy_for_advanced_breast_cancer_in_postmenopausal_women.pdf.

6. Bonneterre, J., Buzdar, A., Nabholtz, J. M., Robertson, J. F., Thürlimann, B. von Euler, M., ...\& Arimidex Writing Committee on behalf of the Investigators Committee Members. (2001). Anastrozole is superior to tamoxifen as first-line therapy in hormone receptor positive advanced breast carcinoma: results of two randomized trials designed for combined analysis. Cancer, 92(9), 2247-2258. doi: 10.1002/10970142 (20011101)92:9<2247::AID-CNCR1570>3.0.CO;2-Y

7. Mouridsen, H., Gershanovich, M., Sun, Y., Pérez-Carrión, R., Boni, C., Monnier, A., ...\& Pluzanska, A. (2001). Superior efficacy of letrozole versus tamoxifen as first-line therapy for postmenopausal women with advanced breast cancer: results of a phase III study of the International Letrozole Breast Cancer Group. Journal of Clinical Oncology, 19(10), 2596-2606. Retrieved from http://citeseerx.ist.psu.edu/viewdoc/download?doi=10.1.1. 466.9741\&rep=rep $1 \&$ type=pdf.

8. Mouridsen, H., Gershanovich, M., Sun, Y., Pérez-Carrión, R., Boni, C. Monnier, A., ...\& Pluzanska, A. (2003). Phase III study of letrozole versus tamoxifen as first-line therapy of advanced breast cancer in postmenopausa women: analysis of survival and update of efficacy from the International Letrozole Breast Cancer Group. Journal of Clinical Oncology, 21(11), 2101-2109. doi: 10.1200/JCO.2003.04.194

9. Paridaens, R. J., Dirix, L. Y., Beex, L. V., Nooij, M., Cameron, D. A. Cufer, T., ...\& Therasse, P. (2008). Phase Ill study comparing exemestane with tamoxifen as first-line hormonal treatment of metastatic breast cancer in postmenopausa women: the European Organisation for Research and Treatment of Cancer Breas Cancer Cooperative Group. Journal of clinical oncology, 26(30), 4883-4890. doi: 10.1200/JCO.2007.14.4659

10. Osborne, C. K., \& Schiff, R. (2005). Estrogen-receptor biology: continuing progress and therapeutic implications. Journal of Clinical Oncology, 23(8), 1616-1622. doi: $10.1200 / J C O .2005 .10 .036$.
11. Jordan, V. C., \& O'Malley, B. W. (2007). Selective estrogen-receptor modulators and antihormonal resistance in breast cancer. Journal of clinical oncology, 25(36), 5815-5824. doi: 10.1200/JCO.2007.11.3886.

12. Osborne, C. K., Wakeling, A., \& Nicholson, R. I. (2004). Fulvestrant: an oestrogen receptor antagonist with a novel mechanism of action. British journal of cancer, 90 (1), S2. https://doi.org/10.1038/sj.bjc.6601629.

13. Kumar, V., Green, S., Stack, G., Berry, M., Jin, J. R., \& Chambon, P. (1987). Functional domains of the human estrogen receptor. Cell, 51(6), 941-951. https://doi.org/10.1016/0092-8674(87)90581-2.

14. Kato, S., Endoh, H., Masuhiro, Y., Kitamoto, T., Uchiyama, S., Sasaki, H., \& Metzger, D. (1995). Activation of the estrogen receptor through phosphorylation by mitogen-activated protein kinase. Science, 270(5241), 1491-1494. doi: 10.1126/science.270.5241.1491.

15. Robertson, J. F. (2007). Fulvestrant (Faslodex ${ }^{\oplus}$ ) - how to make a good drug better. The oncologist, 12(7), 774-784. doi: 10.1634/theoncologist.12-7-774.

16. Wakeling, A. E., Dukes, M., \& Bowler, J. (1991). A potent specific pure antiestrogen with clinical potential. Cancer research, 51(15), 3867-3873. Retrieved from http://cancerres. aacrjournals.org/content/51/15/3867. short.

17. Wakeling, A. E. (2000). Similarities and distinctions in the mode of action of different classes of antioestrogens. Endocrine-related cancer, 7(1), 17-28. Retrieved from https://erc. bioscientifica.com/content/7/1/17.full.pdf

18. Wakeling, A. E. (1995). Use of pure antioestrogens to elucidate the mode of action of oestrogens. Biochemical pharmacology, 49(11), 1545-1549. https://doi.org/10.1016/0006-2952(94)00528-T.

19. Morales, L., Neven, P., Timmerman, D., Wildiers, H., Konstantinovic, M. L., Christiaens, M. R., ...\& Paridaens, R. (2009). Prospective assessment of the endometrium in postmenopausal breast cancer patients treated with fulvestrant. Breast cancer research and treatment, 117(1), 77. https://doi.org/10.1007/s10549-008-0248-2.

20. Peekhaus, N. T., Chang, T., Hayes, E. C., Wilkinson, H. A., Mitra, S. W., Schaeffer, J. M., \& Rohrer, S. P. (2004). Distinct effects of the antiestrogen Faslodex on the stability of estrogen receptors-alpha and-beta in the breast cancer cell line MCF-7. Journal of molecular endocrinology, 32(3), 987-995. https://doi.org/10.1677/jme.0.0320987.

21. Mishra, A. K., Abrahamsson, A., \& Dabrosin, C. (2016). Fulvestrant inhibits growth of triple negative breast cancer and synergizes with tamoxifen in ERa positive breast cancer by up-regulation of ERR. Oncotarget, 7(35), 56876. doi: 10.18632/oncotarget.10871.

22. Deebska-Szmich, S., Ziebba, A., \& Potemski, P. (2017). Fulvestrant in hormonal treatment of breast cancer. Oncology in Clinical Practice, 13(1), 14-23. doi: 10.5603/OCP.2017.0003.

23. McClelland, R. A., Gee, J. M. W., Francis, A. B., Robertson, J. F. R., Blarney, R.W., Wakeling, A. E., \& Nicholson, R. I. (1996). Short-term effects of pure anti-oestrogen ICl 182780 treatment on oestrogen receptor, epidermal growth factor receptor and transforming growth factor-alpha protein expression in human breast cancer. European Journal of Cancer, 32(3), 413-416. https://doi.org/10.1016/0959-8049(95)00517-X.

24. Inoue, A., Yoshida, N., Omoto, Y., Oguchi, S., Yamori, T., Kiyama, R., \& Hayashi, S. (2002). Development of cDNA microarray for expression profiling of estrogen responsive genes. Journal of Molecular Endocrinology, 29(2), 175192. http://citeseerx.ist.psu.edu/viewdoc/download?doi=10.1.1.887.2620\&rep rep $1 \&$ type=pdf

25. Garcia Dos Santos, E., Dieudonne, M. N., Pecquery, R., Le Moal, V., Giudicelli, Y., \& Lacasa, D. (2002). Rapid nongenomic E2 effects on p42/p44 MAPK, activator protein-1, and cAMP response element binding protein in rat white adipocytes. Endocrinology, 143(3), 930-940. https://doi.org/10.1210/endo.143.3.8678.

26. Hu, X. F., Veroni, M., De Luise, M., Wakeling, A., Sutherland, R. Watts, C. K. W., \& Zalcberg, J. R. (1993). Circumvention of tamoxifen resistance by the pure anti-estrogen ICI 182, 780. International journal of cancer, 55(5), 873-876. https://doi.org/10.1002/ijc.2910550529.

27. Lykkesfeldt, A. E., Madsen, M. W., \& Briand, P. (1994). Altered expression of estrogen-regulated genes in a tamoxifen-resistant and $\mathrm{ICI} 164,384$ and ICl 182,780 sensitive human breast cancer cell line, MCF-7/TAMR-1. Cancer research, 54(6), 1587-1595. https://cancerres.aacrjournals.org/content/54/6/1587. short.

28. de Cupis, A., Noonan, D., Pirani, P., Ferrera, A., Clerico, L., \& Favoni, R. E. (1995). Comparison between novel steroid-like and conventional nonsteroidal antioestrogens in inhibiting oestradiol-and IGF-I-induced proliferation of human breast cancer-derived cells. British journal of pharmacology, 116(5), 2391-2400. https://doi.org/10.1111/j.1476-5381.1995.tb15085.x.

29. Lykkesfeldt, A. E., Larsen, S. S., \& Briand, P. (1995). Human breast cancer cell lines resistant to pure anti-estrogens are sensitive to tamoxifen treatment. International journal of cancer, 61(4), 529-534. https://doi.org/10.1002/ijc.2910610417.

30. Osborne, C. K., Coronado-Heinsohn, E. B., Hilsenbeck, S. G., McCue, B. L., Wakeling, A. E., McCleland, R. A., ...\& Nicholson, R. I. (1995). Comparison of the effects of a pure steroidal antiestrogen with those of tamoxifen in a model of human breast cancer. Journal of the National Cancer Institute, 87(10), 746-750. https://doi.org/10.1093/jnci/87.10.746.

31. Dowsett, M., Nicholson, R. I., \& Pietras, R. J. (2005). Biological characteristics of the pure antiestrogen fulvestrant: overcoming endocrine resistance. Breast cancer research and treatment, 93(1), 11-18. doi: https://doi.org/10.1007/s10549-005-9037-3.

32. Rocca, A., Maltoni, R., Bravaccini, S., Donati, C., \& Andreis, D. (2018). Clinical utility of fulvestrant in the treatment of breast cancer: a report on the emerging clinical evidence. Cancer management and research, 10, 3083. doi: 10.2147/CMAR.S137772.

33. Howell, A., Robertson, J. F., Quaresma Albano, J., Aschermannova, A., Mauriac, L., Kleeberg, U. R., ...\& Morris, C. (2002). Fulvestrant, formerly ICI 182,780, is as effective as anastrozole in postmenopausal women with advanced breast cancer progressing after prior endocrine treatment. Journal of Clinical Oncology, 20(16), 3396-3403. doi: 10.1200/JCO.2002.10.057.

34. Osborne, C. K., Pippen, J., Jones, S. E., Parker, L. M., Ellis, M., Come, S., ..\& Webster, A. (2002). Double-blind, randomized trial comparing the efficacy and tolerability of fulvestrant versus anastrozole in postmenopausal women with advanced breast cancer progressing on prior endocrine therapy: results of a North American trial Journal of Clinical Oncology, 20(16), 3386-3395. doi: 10.1200/JCO.2002.10.058.

35. Robertson, J. F., Nicholson, R. I., Bundred, N. J., Anderson, E., Rayter, Z., Dowsett, M., ...\& Morris, C. (2001). Comparison of the short-term biological effects of $7 \alpha-[9-(4,4,5,5,5$-pentafluoropentylsulfinyl)-nonyl] estra-1, 3 , $5,(10)$-triene-3, 17 $\beta$-diol (Faslodex) versus tamoxifen in postmenopausal women with primary breast cancer. Cancer research, 61(18), 6739-6746. Retrieved from http://cancerres.aacrjournals.org/content/61/18/6739.short. 
36. DeFriend, D. J., Howell, A., Nicholson, R. I., Anderson, E., Dowsett, M. Mansel, R. E., ...\& Baum, M. (1994). Investigation of a new pure antiestrogen (ICI 182780) in women with primary breast cancer. Cancer Research, 54(2), 408-414. Retrieved from http://cancerres.aacrjournals.org/content/54/2/408.short.

37. Howell, A., DeFriend, D. J., Robertson, J. F. R., Blamey, R. W., Anderson, L., Anderson, E., ...\&Walton, P. (1996). Pharmacokinetics, pharmacological and anti-tumour effects of the specific anti-oestrogen ICI 182780 in women with advanced breast cancer. British Journal of Cancer, 74(2), 300. https://doi.org/10.1038/bjc.1996.357.

38. AstraZeneca. Faslodex (fulvestrant) injection, for intramuscular use US prescribing information. 2017. https://www.accessdata.fda.gov. Accessed 11 Dec 2017

39. AstraZeneca UK Limited. Faslodex $250 \mathrm{mg}$ solution for injection: EU summary of product characteristics. 2017. http://www.ema.europa.eu/ema. Accessed 11 Dec 2017.

40. Belagali, Y. R., Barkate, H. V., Sejpal, J. J., \& Parekh, B. B. (2016). Therapeutic place of fulvestrant in the management of hormone-receptor positive breast cancer. Eur Oncol Haematol, 12(1), 44-50. http://doi.org/10.17925/EOH.2016.12.01.44.

41. Deeks, E. D. (2018). Fulvestrant: a review in advanced breas cancer not previously treated with endocrine therapy. Drugs, 78(1), 131-137. https://doi.org/10.1007/s40265-017-0855-5.

42. Cardoso, F., Costa, A., Senkus, E., Aapro, M., André, F., Barrios, C. H., ... \& Carey, L. (2017). 3rd ESO-ESMO international consensus guidelines fo advanced breast cancer (ABC 3). Annals of Oncology, 28(1), 16-33. https://doi.org/10.1093/annonc/mdw544.

43. National Comprehensive Cancer Network. NCCN clinical practice guidelines in oncology (NCCN Guidelines). Breast cancer version 3.2017. 2017. http://www.nccn.org. Accessed 11 Dec 2017.

44. Robert, N. J., \& Denduluri, N. (2018). Patient case lessons: endocrine management of advanced breast cancer. Clinical breast cancer, 18(3), 192-204. https://doi.org/10.1016/j.clbc.2017.05.014.

45. Boér, K. (2017). Fulvestrant in advanced breast cancer: evidence to date and place in therapy. Therapeutic advances in medical oncology, 9(7), 465-479. https://doi.org/10.1177/1758834017711097.

46. Telford, C., Takyar, S., Joshi, P., Ekman, M., \& Jones, N. (2017). A network meta-analysis of fulvestrant vs alternative first-line endocrine therapies for endocrine therapy-naive postmenopausal hormone receptor-positive advanced or metastatic breast cancer. doi: 10.1200/JCO.2017.35.15_suppl.e12545.

47. Zhang, J., Huang, Y., Wang, C., He, Y., Zheng, S., \& Wu, K. (2017). Efficacy and safety of endocrine monotherapy as first-line treatment for hormonesensitive advanced breast cancer: a network meta-analysis. Medicine, 96(33). doi 10.1097/MD.0000000000007846.

48. Інструкція для медичного застосування лікарського засобу № UA/5440/01/01 від 19.07.2016 до 19.07.2021.

49. Di Leo, A., Jerusalem, G., Petruzelka, L., Torres, R., Bondarenko, I. N., Khasanov, R., ... \& Pendergrass, K. (2010). Results of the CONFIRM phase III trial comparing fulvestrant $250 \mathrm{mg}$ with fulvestrant $500 \mathrm{mg}$ in postmenopausal women with estrogen receptor-positive advanced breast cancer. J Clin Oncol, 28(30), 4594-4600. doi: 10.1200/JCO.2010.30.6266
50. Robertson, J. F., Llombart-Cussac, A., Rolski, J., Feltl, D., Dewar, J., Macpherson, E., ...\& Ellis, M. J. (2009). Activity of fulvestrant $500 \mathrm{mg}$ versus anastrozole $1 \mathrm{mg}$ as first-line treatment for advanced breast cancer: results from the FIRST study. Journal of Clinical Oncology, 27(27), 4530-4535. doi: 10.1200/JCO.2008.21.1136.

51. Robertson, J. F., Lindemann, J. P., Llombart-Cussac, A., Rolski, J., Feltl, D., Dewar, J., ...\& Ellis, M. J. (2012). Fulvestrant $500 \mathrm{mg}$ versus anastrozole $1 \mathrm{mg}$ for the first-line treatment of advanced breast cancer: follow-up analysis from the randomized «FIRST» study. Breast cancer research and treatment, 136(2), 503-511. doi: https://doi.org/10.1007/s10549-012-2192-4.

52. Ellis, M. J., Llombart-Cussac, A., Feltl, D., Dewar, J. A., Jasiówka, M., Hewson, N., ...\& Robertson, J. F. (2015). Fulvestrant $500 \mathrm{mg}$ versus anastrozole $1 \mathrm{mg}$ for the firstline treatment of advanced breast cancer: overall survival analysis from the phase II FIRST study. Journal of Clinical Oncology, 33(32), 3781. doi: 10.1200/JCO.2015.61.5831.

53. Howell, A., Robertson, J. F., Quaresma Albano, J., Aschermannova, A., Mauriac, L., Kleeberg, U. R., ...\& Morris, C. (2002). Fulvestrant, formerly ICI 182,780, is as effective as anastrozole in postmenopausal women with advanced breast cancer progressing after prior endocrine treatment. Journal of Clinical Oncology, 20(16), 3396-3403. doi: 10.1200/JCO.2002.10.057.

54. Robertson, J. F., Osborne, C. K., Howell, A., Jones, S. E., Mauriac, L., Ellis, M. ...\& Buzdar, A. (2003). Fulvestrant versus anastrozole for the treatment of advanced breast carcinoma in postmenopausal women: a prospective combined analysis of two multicenter trials. Cancer, 98(2), 229-238. doi: 10.1002/cncr.11468.

55. Kuter, I., Hegg, R., Singer, C., Badwe, R., \& Lowe, E. (2007). Fulvestrant $500 \mathrm{mg}$ vs $250 \mathrm{mg}$ : first results from NEWEST, a randomized, phase II neoadjuvant trial in postmenopausal women with locally advanced, estrogen receptor-positive breast cancer. Breast Cancer Research and Treatment, 106. doi: 10.1007/s10549-007-9793-3.

56. Di Leo, A., Jerusalem, G., Petruzelka, L., Torres, R., Bondarenko, I. N., Khasanov, R., ...\& Pendergrass, K. (2012). Abstract S1-4: Final analysis of overall survival for the Phase III CONFIRM trial: fulvestrant $500 \mathrm{mg}$ versus $250 \mathrm{mg}$. doi: 10.1158/0008-5472.SABCS12-S1-4.

57. Leo, A. D., Jerusalem, G., Petruzelka, L., Torres, R., Bondarenko, I. N., Khasanov, R ... \& Pendergrass, K. (2013). Final overall survival: fulvestrant $500 \mathrm{mg}$ vs $250 \mathrm{mg}$ in the randomized CONFIRM trial. Journal of the National Cancer Institute, 106(1), djt337. doi: 10.1093/jnci/djt337.

58. Nabholtz, J. M., Bonneterre, J., Buzdar, A., Robertson, J. F. R., Thürlimann, B. (2003). Anastrozole (Arimidex ${ }^{\mathrm{TM}}$ ) versus tamoxifen as first-line therapy for advanced breast cancer in postmenopausal women: survival analysis and updated safety results. European Journal of Cancer 39(12), 1684-1689 doi: 10.1016/S0959-8049(03)00326-5.

59. Mehta, R. S., Barlow, W. E., Albain, K. S., Vandenberg, T. A., Dakhil, S. R., Tirumali, N. R., ...\& Hortobagyi, G. N. (2012). Combination anastrozole and fulvestrant in metastatic breast cancer. New England Journal of Medicine, 367(5), 435-444. doi: 10.1056/NEJMoa1201622.

60. Robertson, J. F., Bondarenko, I. M., Trishkina, E., Dvorkin, M., Panasci, L., Manikhas, A., ...\& Ruiz-Borrego, M. (2016). Fulvestrant 500 mg versus anastrozole $1 \mathrm{mg}$ for hormone receptor-positive advanced breastcancer (FALCON): an international, randomised, double-blind, phase 3 trial. The Lancet, 388(10063), 2997-3005. doi: 10.1016/S0140-6736(16)32389-3.

Евгения Гониарук 